\title{
Comparative Phylogenomic Analysis of Cytochrome P450 Monooxygenases From Fusarium Species
}

Wadzani Palnam Dauda ( $\nabla$ wadzanidauda@gmail.com )

Federal University, Gashua https://orcid.org/0000-0002-5233-0058

Elkanah Glen

Federal University Lokoja

Peter Abraham

Federal College of Horticulture

Charles Oluwaseun Adetunji

Edo University lyamho

\section{Daji Morumda}

Federal University Wukari

Shittu Emmanuel Abraham

Bayero University Kano

Grace Peter Wabba

Ahmadu Bello University

Israel Ogra Ogwuche

Ahmadu Bello University

Mawuli K. Azameti

CSIR-Food Research Institute

\section{Research Article}

Keywords: Cytochrome P450, Evolutionary, Fusarium, Metabolites, Mycotoxin

Posted Date: December 2nd, 2021

DOI: https://doi.org/10.21203/rs.3.rs-1097665/v1

License: (c) This work is licensed under a Creative Commons Attribution 4.0 International License. Read Full License 


\section{Abstract}

Cytochrome P450s (P450s) are a unique multifamily class of enzymes that possess the capability to exhibit catalytic versatility in several biochemical reactions which entails metabolite biosynthesis, primary and secondary metabolism. Fusarium spp. is an important microorganism with many members known to produce secondary metabolites that cause plant diseases and mycotoxicoses in animals and humans. In this present study, from the initially screened 4,579 proteins, we elucidated the nature of abundance, evolutionary relationships, classification and cellular location of 320 cytochrome P450 from 17 phytopathogenic members of Fusarium species. The total CYPs protein sequences were phylogenetically grouped into seventeen (17) clades. Eighty-six (86) CYPs families and forty-eight (48) clans were identified. Twenty-seven (27) families were each found in only one species. The CYPs were found to be majorly localized in the endoplasmic reticulum. The non-ribosomal peptide synthetase-like (NRPS-like) gene cluster was the predominant secondary metabolicrelated gene cluster across all the seventeen selected Fusarium species except in F. cucurbiticola and F. solani, where PolyKetide Synthase (PKS) was the most prevalent. The presence of numerous families and clans as observed in in this study shows the expansions of the CYPs families across Fusarium species, this CYPs family and clan expansion is often associated with the evolvement of several fungal traits that include their pathogenicity adaptation to survive on an extensive range of toxic substrates. Identification of P450 proteins in these pathogenic fungi provides fundamental information for further basic and applied biological research into the physiological and toxigenic roles of P450s in Fusarium species.

\section{Introduction}

Fusarium species are ubiquitous economically important fungi of the filamentous ascomycete. They produce many diverse plants pathogenic toxicants that cause blights, wilts, cankers and rots, among many other diseases (Ma et al., 2013). The damaging effects of Fusarium mycotoxins on plants have been reported to be greater than any known contaminant or cluster of toxins (Munkvold, 2017). Fusarium genera have been reported to produce secondary metabolites that are very toxic and are known to cause diverse diseases in plants and mycotoxicoses in both animals and humans (Munkvold et al., 2021, Ezrari et al., 2021). The vast spread of Fusarium species might be a function of its ability to survive on an extensive range of toxic substrates as well as various pollutants via their cytochrome P450 enzymes which can degrade completely harmful substances and still produce secondary metabolites that are toxic to serve as weapons against other competitors that include plants, animals, bacteria and other fungi in a limited ecological niche (Shin et al., 2018).

Typical examples of these secondary metabolites which are myotoxic in nature include trichothecenes, moniliformins, zearalenone, and fumonisins, which all have the potential to affect agricultural produce and contaminate food/feed, consequently leading to animal diseases, defects of neural tube and cancer in humans (Proctor et al., 2016). Fusarium genera are generally known to affect agriculturally important crops such as maize, coffee, rice, banana, wheat, citrus, mango and sugar cane. (Proctor et al., 2016). Munkvold et al. (2021) observed that members of the Fusarium species complexes are collectively known to cause various diseases among diverse economically important plants, hence causing substantial yield losses at pre/post-harvest and at the cause of storage, contaminating food and aggravating food security (Seepe et al., 2020).

Cytochrome P450 (CYPs) are cosmopolitan group of enzymes that have the potential to either cause/prevent toxicity by transforming substances into reactive products that cause toxicity or by oxidizing them to prevents toxicity respectively (Guengerich, 2020). CYPs have been implicated in the degradation of diverse arrays of substances that are of both exogenous and endogenous origin primarily due to their ability to facilitate the oxidation of enormous substrate with minimal reaction conditions, hence enabling them to play active roles in the degradation of xenobiotics, performs primary and secondary metabolism which are important to the survival of the organisms (Chadha et al., 2018). Fungal CYP enzymes have been reported to be responsible for key roles in the biosynthesis of numerous secondary metabolites of 
great importance to the industrial, agricultural and biomedical settings (Durairaj et al., 2015). Cytochrome P450s derived from microbes are of great interest because they act as fundamental elements in bioremediation and the formation of natural products in microbes. They are principal targets for agrochemical and drugs and serve as biocatalysts (Durairaj et al., 2015). Some individual Fusaria have been studied, and information on their secondary metabolic-related gene clusters are available like Fusarium fujikuroi, F. lycopersici and F. verticilloides. Several mycotoxins have been identified and reported to cause diseases in many plants and animals. To the best of our knowledge, a comprehensive comparative study into the cytochrome P450 enzymes involving these agriculturally important fungi (Fusarium species) with potential effects on animals and humans have not been carried out. Therefore, this study intends to look at the diversity and similarities of the CYPs across some selected agriculturally important Fusarium species in order to evaluate their evolutionary relationship with the view to provide information on possible general or specific approaches to mitigating the economic, health and environmental loss due to the pathogenic complexities and ubiquitous nature of these fungi.

\section{Methods}

\section{Species Selection}

There are about sixty (60) different species of Fusarium that have been deposited in the Mycocosm of the joint genome institute as of August 2021. Seventeen have been selected for this study and they include; Fusarium oxysporum $f . s p$. conglutinans race 254008 (PHW808), Fusarium oxysporum f. sp. cubense tropical race 454006 (II5), Fusarium cucurbiticola FSSC 10 v1.0, Fusarium fujikuroi IMI 58289, Fusarium graminearum v1.0, Fusarium langsethiae FI201059, Fusarium oxysporum f. sp. lycopersici MN25 (FoMN25) NRRL 54003, Fusarium mangiferae MRC7560, Fusarium oxysporum Fo5176 V1.0, Fusarium oxysporum f. sp. pisi HDV247, Fusarium poae 2516, Fusarium proliferatum ET1, Fusarium pseudograminearum CS3096, Fusarium solani FSSC 5 v1.0, Fusarium oxysporum f. sp. vasinfectum 25433 (Cotton), Fusarium verticillioides 7600 v2.

The selection was based on the reported damaging effects of these species in agriculturally important crops such as maize, cotton, sugar cane, soybeans. Fusarium oxysporum f. sp. Lycopersici is specific to tomato, Fusarium verticillioides targets sorghum and maize but can still infect other plants, Fusarium mangiferae causes mango malformation (Niehaus et al., 2016), Fusarium langsethiae affects oats, wheat and barley kernels (Lysoe et al., 2016), Fusarium proliferatum infects garlic and asparagus, Fusarium oxysporum 5176 is pathogenic to roots of most plants (Fokkens et al., 2020).

\section{Sequence Retrieval}

A total of four thousand five hundred and seventy-nine $(4,579)$ cytochrome P450 protein FASTA sequences from the seventeen (17) species of Fusarium spp were downloaded from the Joint Genome Institute server. https://mycocosm.jgi.doe.gov/mycocosm/home.

\section{Validation/screening Of Sequences}

The downloaded sequences $(4,579)$ were first screened for cytochrome P450 conserved domain from the fungal cytochrome P450 database, where 2701 sequences were found to have P450 conserved domain from the fungal cytochrome P450 database as shown in Table 1. These were further subjected for validation using the NCBI batch conserved domain database tool at a stringent e-value of $\geq 7$ (Chadha et al., 2018). A total of 320 sequences met this condition and were used for other analyses.

Evolutionary Relationship of Cytochrome P450 Proteins Across Seventeen Fusarium species: 
The 320 downloaded protein sequences were subjected to multiple sequence alignment using the MUSCLE algorithm in MEGA X software with alignment options as follows; Gap open: -2.90, Gap extend 0.00, hydrophobicity multiplier: 1.20, Max Memory in MB:2048, Max iterations:16, Cluster method: UPGMA and Min Diag. Length (Lambda): 24. After the MUSCLE alignment, the phylogenetic tree was constructed using the neighbour-joining method and Jones-Taylor-Thornton (JTT) model, uniform rates among sites and pairwise deletion gaps treatment were used (http://www.megasoftware. net/).

Identification of Cytochrome P450 families and clans in seventeen Fusarium species:

Cytochrome P450 families were identified by blasting the sequences against known fungal cytochrome P450 in the fungal cytochrome P450 database (http://p450.riceblast.snu.ac.kr/blast.php) percentage similarity of each query sequence was compared against the known sequences that have been characterized, any query sequence with percentage similarity of greater than 40 is placed in the same family based on the method of international P450 nomenclature committee (Chadha et al., 2018). Corresponding Clans were allocated based on the classification of families into clans in the database (http://p450.riceblast.snu.ac.kr/clans.php).

\section{Subcellular Localization Prediction Of Protein Genes}

The subcellular localization prediction of all the protein genes across the seventeen Fusarium species was performed with the aid of an online server for predicting subcellular localization of protein with single or multiple sites iLoc-Animal (http://www.jci-bioinfo.cn/iLoc-Animal)

\section{Secondary Metabolic Related Gene Clusters}

The secondary metabolic-related gene clusters were retrieved from Mycocosm (https://mycocosm.jgi.doe.gov/mycocosm/home) using the annotation menu and compiled for the seventeen selected Fusarium spp.

\section{Results}

\section{Cyp Proteins In Fusarium:}

The highlights of the taxonomic distribution of the putative cyps of the 320 selected protein sequences from the selected Fusarium species (Table 1), shows F. pisi has the highest protein entry of 34, followed by F. oxysporum 5176 with 32 entries, on the other hand, F. cucurbiticola has the least protein entry of 4. F.oxysporum Fo5176 and F, melonis are the two species that have some families with no matches in the fungal cytochrome P450 database. F. oxysporum Fo5176 has 13 families while F. melonis has 3 families with no matches in the fungal cytochrome P450 database.

\section{Evolutionary Relationship Of Taxa}

The phylogenetic analysis of the P450s present in the seventeen selected Fusarium species (Figure 1) revealed the presence of several branches, which suggest a strong evolutionary divergence over time. CYPs belonging to the same family irrespective of the Fusarium species were clustered on the same monophyletic clade on the phylogenetic tree. The CYPs across all the selected species were grouped into seventeen (17) clades based on their phylogenetic relationship. Clades III and IX have the highest branches with a total P450 of 33 and 37, respectively. The lowest number of branches (6 CYPs) was recorded in clade XVI. Clan CYP531 was the most expanded with members across four clades (IV, IX, X and 
XIV). Eighteen (CYPs) with no matches in the fungal cytochrome P450 database (FCDP) for family classification were found to be clustered in just four clades; four (4) each in clades VI and VII, while five (5) each in clades VIII and IX. Based on the phylogenetic clustering, three (3) of these unmatched CYPs from Fusarium oxysporum melonis were classified alongside CYP61 (clade VIII), CYP5085 in clade IX and clan CYP61 in clade VI. Moreover, the result obtained from Fusarium oxysporum 5176, three (3) CYPs were closely related to CYP61 (clade VII), four (4) with CYP505 and one (1) CYP645 in clades VIII, also one (1) with CYP5111 and three (3) with CYP5085 in clades IX, finally, one (1) with CYP61 and two (2) with CYP61/CYP621/CYP637.

The phylogenetic clustering of these CYPs across the seventeen Fusarium species is shown in Table 2; All the selected species were represented in clade $V$ except for F. cucurbiticola, clade I has CYPs from 14 species out of the seventeen selected species, clades III, VI, IX, XII and IV have CYPs from 13 selected species each. Clades with the least number of species are; $\mathrm{XVI}, \mathrm{XV}, \mathrm{XIII}$ and $\mathrm{VIII}$ with $4,5,6$ and 6 respectively.

All the species selected were found to have CYPs that spread across at least nine (9) clades out of the seventeen identified clades except F. cucurbiticola and F. graminearum that were found to appear in just 2 and 6 clades respectively (Table 2). 
Table 1

Taxonomic Distributions of Cytochrome P450 in Seventeen (17) Fusarium spp.

\begin{tabular}{|c|c|c|c|c|c|c|c|c|}
\hline Fusarium Species & $\begin{array}{l}\text { Genome } \\
\text { size }(\mathrm{Mb})\end{array}$ & $\begin{array}{l}\text { Number } \\
\text { of } \\
\text { predicted } \\
\text { genes }\end{array}$ & $\begin{array}{l}\text { Total } \\
\text { proteins } \\
\text { Sequences }\end{array}$ & $\begin{array}{l}\text { Total } \\
\text { Cyp } \\
\text { proteins }\end{array}$ & $\begin{array}{l}\text { Sequences } \\
\text { with } e \\
\text { value } \geq 7\end{array}$ & $\begin{array}{l}\text { Family } \\
\text { type }\end{array}$ & $\begin{array}{l}\text { Clan } \\
\text { type }\end{array}$ & $\begin{array}{l}\text { Families } \\
\text { with no } \\
\text { FCPD } \\
\text { matches }\end{array}$ \\
\hline $\begin{array}{l}\text { F. oxysporum } \\
\text { Fo5176 }\end{array}$ & $67,983,296$ & 19,130 & 297 & 185 & 32 & 16 & 15 & 13 \\
\hline F.o. melonis & $54,034,280$ & 26,719 & 344 & 201 & 21 & 13 & 13 & 3 \\
\hline F.o. conglutinans & $53,575,352$ & 26,246 & 345 & 210 & 20 & 13 & 12 & - \\
\hline F.o. lycopersici & $48,637,398$ & 24,733 & 319 & 189 & 24 & 21 & 18 & - \\
\hline F.o. vasinfectum & $52,914,414$ & 25,216 & 320 & 196 & 21 & 18 & 13 & - \\
\hline F.o.pisi & $55,188,216$ & 26,378 & 355 & 217 & 34 & 25 & 21 & - \\
\hline F.o.cubense & $46,553,780$ & 22,487 & 293 & 172 & 16 & 14 & 14 & - \\
\hline F. verticillioides & $41,776,161$ & 20,553 & 288 & 148 & 19 & 17 & 15 & - \\
\hline F. fujikuroi & $43,832,314$ & 14,813 & 276 & 143 & 13 & 11 & 9 & - \\
\hline $\begin{array}{l}F \\
\text { pseudograminearum }\end{array}$ & $36,330,246$ & 12,395 & 173 & 108 & 17 & 16 & 13 & - \\
\hline F. graminearum & $36,446,046$ & 13,322 & 209 & 110 & 9 & 8 & 7 & - \\
\hline F. mangiferae & $46,292,722$ & 15,804 & 261 & 158 & 20 & 20 & 17 & - \\
\hline F. langsethiae & $37,543,021$ & 11,940 & 184 & 119 & 17 & 16 & 14 & - \\
\hline F. poae & $46,476,831$ & 14,740 & 201 & 125 & 18 & 18 & 18 & - \\
\hline F. proliferatum & $45,210,324$ & 16,143 & 285 & 181 & 20 & 19 & 14 & - \\
\hline F.solani & $52,932,111$ & 17,656 & 279 & 158 & 15 & 13 & 12 & - \\
\hline F. cucurbiticola & $42,448,309$ & 12,147 & 150 & 81 & 4 & 4 & 4 & - \\
\hline Total & & & 4,579 & 2,701 & 320 & & & \\
\hline
\end{tabular}

\section{Families And Clan Classification}

A total of eighty-six (86) Cyp families and forty-eight (48) CYP clans were identified across the seventeen (17) selected Fusarium species as shown in figure 2. Twenty-seven (27) of these Cyp families were found to be unique to only a specie. Fusarium oxysporum 5176 has five (5) families unique to it alone (not found in other selected species). Other families unique to only a single specie out of the selected species are; Cyp506 and Cyp5111 are unique to F. conglutinans, Cyp504 in F.cucurbiticola, Cyp 5156 was found only in F. lycopersici, Cyp633 only in F. lansetheaie, Cyp636 in F.melonis, Cyp503, Cyp530, and Cyp573 in F.o. pisi, Cyp62, Cyp631 and Cyp673 were found only in F.poae, Cyp547, Cyp622, Cyp5078, Cyp5104, Cyp640, in F.O. 5176, Cyp644 and Cyp540 only in F. graminearum, Cyp 629 only in F. vasifectum, Cyp587 and Cyp625 in F. verticilloides, Cyp583 in F. proliferatum, Cyp534 in F. pseudograminearum, Cyp675, Cyp683 and Cyp5109 were found only in F. solani. These twenty-seven unique families were spread across thirteen Fusarium species. The largest clan (both in terms of the number of P450s and number of families) is CYP531, with 17 P450s and eight (8) different families (Figure 2). The most consolidated clan is CYP505, with 16 P450s all belonging to one family. 


\section{Subcellular Localization}

The distribution of the CYPs across the subcellular organelles was found to be majorly localized in the endoplasmic reticulum, as seen in (Figure 3a). In contrast, very few sequences were found scattered across the other organelles except for $F$. oxysporum 5176 , which has 8 cyps localized in the cytoplasm after the major localization in the endoplasmic reticulum (Figure $3 b$ ).

\section{The Distribution of Secondary Metabolic Related Gene Clusters}

The secondary metabolic-related gene clusters of CYPs present in the selected Fusarium species were presented in Figure 4a. The non-ribosomal peptide synthetase-like (NRPS-like) gene cluster was the predominant gene cluster across all the seventeen selected Fusarium species except in F. cucurbiticola and F. solani, which have PolyKetide Synthase (PKS) as the predominant gene clusters. Figure $4 \mathrm{~b}$ showed the distribution of all the secondary metabolism gene clusters in each specie. F. cucurbiticola and F. lansatheiae have no HYBRID gene clusters, like-wise F. graminearum, F. poae and $F$. pseudograminearum contain no DMATS.

\section{Discussion}

The phyletic studies have shown that the distribution of Cytochrome P450 across the seventeen (17) clades varies in size. This is connected with the various narrowing and enlargements of some families of CYP through the path of evolution. The gain of some CYP clans in certain Fusarium species contributes to the survival ability of these species over extreme conditions in their habitat. This agrees with the earlier submission by (Shin et al., 2018), where they attributed the ubiquitous nature of fungi to their ability to use cytochrome P450 enzymes in adapting to diverse environmental stress and quickly adjust to harsh ecological niches, consequently the widespread of these fungi in decaying materials (organic), soil, animals and living plants. Study revealed that particular P450 families in some fungi species are enhanced by paralogous evolution of member P450s to enable the organism adapt to varied ecological niches, such as plant material colonization. (Syed et al., 2014). In other words, P450 family expansion is possible due to the duplication of member P450s in an organism (Sello et al., 2015; Feyereisen, 2011).

The presence of numerous families and clans in the seventeen selected Fusarium species indicates the expansions of the fungal CYPs families which have been earlier reported Qhanya et al., (2015) to be associated with the evolvement of several fungal traits that include their pathogenicity. The abundance of some unique families to certain species of Fusarium has also collaborated on the existing influence of the interaction of a host and its ecological niches on the contents of CYPs of an organism (Qhanya et al., 2015). Therefore, unique CYPs across several species of Fusarium might be responsible for the host specificity of individual Fusarium species to specific plant or animal as earlier reported (Rampersad, 2020).

The existence of multiple P450s in the selected species, as well as the expansion of specific P450 families and the presence of separate P450 families, suggests that P450s play an important role in these organisms.

Functional analysis for some of these identified P450s can be expected based on homologous P450s identified in other fungal species. For instance, the CYP61 family catalyzes C-22 sterol desaturase activity, which is implicated in membrane ergosterol production (Kelly et al., 1997), while members of the CYP505 family are engaged in the oxidation of fatty acids (Nakayama et al., 1996), and in the production of the mycotoxin fumonisin (Proctor et al., 2003).

The observed expansion of cytochrome P450 families as seen by the occurrence of certain Cyp families in more than five species across the seventeen selected Fusarium species has been related to the functional diversity and emergence of pathogenicity in fungi (Minerdi et al., 2019). Cytochrome P450 has been reported to be very helpful in the functioning of cells where some were found to be involved in the primary metabolism of the cells. Others are specifically responsible for 
the metabolism of xenobiotics, secondary metabolites and defense (Chadha et al., 2018). Several cytochrome P450 have been reported to be linked with pathogen virulence due to their ability to neutralize antibiotics produced by the host. The expansion of these CYPs as observed in this study will not be unconnected with the important roles of these enzymes during the transformation of foreign toxicants such as xenobiotics, toxins derived from plants and environmental pollutants into less toxic ones via the cytochrome P450-mediated mechanism in collaboration with their vibrant extracellular defense system (Shin et al., 2018; Minerdi et al., 2019).

The distribution of secondary metabolic related gene clusters across the selected Fusarium species has shown NRPS-like and PKS as the most abundant gene clusters for secondary metabolism. These abundant gene clusters have been reported to be active in building the general scaffold structure of the majority of the secondary metabolites. Secondary metabolites have generally been reported to be responsible for the pathogenesis of several diseases (Saha et al., 2021). The secondary metabolites associated with fungi include; terpenes, non-ribosomal peptides, polyketides and indole terpenes (Saha et al., 2021). The synthesis of secondary metabolites in fungi is aided by some structural genes that include; P450monoxygenases, methyltransferases, reductases, dimethylallyl tryptophan synthase (DMATS), nonribosomal peptide synthetases (NRPS), non-ribosomal peptide synthetases-LIKE (NRPS-LIKE), polyketide synthases (PKS), polyketide synthase-like (PKS-LIKE) and terpene cyclases (TC) (Saha et al., 2021). The polymerization of the primary metabolites synthesizes these secondary metabolites by backbone enzymes for instance, polyketide synthases(PKS) uses acyl-CoAs to generate polyketides while non-ribosomal peptide synthetases produce non-ribosomal peptides from amino acid (Keller, 2019). Many fungal CYPs are actively involved in mycotoxins biosynthesis, these mycotoxins are the secondary metabolites produced by fungi, mostly in contaminated crops, and they cause severe consequences for animals and humans. Examples of these toxic mycotoxins include trichothecene from F. graminearum which inhibits protein synthesis and exhibits cytotoxicity to several eukaryotes. Similarly, fumonisin from F.verticilliodes is a potent inducer of several diseases in animals such as leukoencephalomalacia, cancer and pulmonary oedema (Shin et al., 2018).

\section{Conclusion}

The study showed the presence of several P450s branches on the phylogenetic tree, which suggests a robust evolutionary divergence over time in the seventeen selected Fusarium species. CYPs from different Fusarium species but the same families were clustered on the same monophyletic clade on the phylogenetic tree. The study also revealed numerous families and clans (eighty-six (86) families and forty-eight (48) clans) in the seventeen selected Fusarium species which implies the expansions of the fungal CYPs families, which are associated with the evolvement of several fungal traits that include their pathogenicity. The abundance of some unique families to certain species of Fusarium shows the influence of an interaction between a host and its ecological niches on the contents of the Fusarium CYPs. Also such unique families may be responsible for the host specificity of individual Fusarium species. The study further showed that the cyps across the selected Fusarium species were majorly localized in the endoplasmic reticulum. The non-ribosomal peptide synthetase-like (NRPS-like) gene cluster was the dominant gene cluster among all the seventeen selected Fusarium species except in F. cucurbiticola and F. solani, which have PolyKetide Synthase (PKS) as the dominant gene clusters. The findings of the present study elucidated some relevant information that could be harnessed for the effective management of Fusarium species which pose a threat to the profitable production and food security of several important crops globally.

\section{References}

1. Chadha, S., Mehetre, S. T., Bansal, R., Kuo, A., Aerts, A., Grigoriev, I. V., Druzhinina, I. S., \& Mukherjee, P. K. (2018). Genome-wide analysis of cytochrome p450s of trichoderma spp.: Annotation and evolutionary relationships. Fungal Biology and Biotechnology, 5(1), 1-15. https://doi.org/10.1186/s40694-018-0056-3

2. Durairaj, P., Malla, S., Nadarajan, S. P., Lee, P., Jung, E., Park, H. H., Kim, B., \& Yun, H. (2015). Fungal cytochrome P450 monooxygenases of Fusarium oxysporum for the synthesis of $\omega$-hydroxy fatty acids in engineered Saccharomyces 
cerevisiae. 1-16. https://doi.org/10.1186/s12934-015-0228-2

3. Ezrari, S., Lahlali, R., Radouane, N., Tahiri, A., Asfers, A., Boughalleb-M'Hamdi, N., Amiri, S., \& Lazraq, A. (2021). Characterization of Fusarium species causing dry root rot disease of citrus trees in Morocco. Journal of Plant Diseases and Protection, 128(2), 431-447. https://doi.org/10.1007/s41348-020-00392-0

4. Feyereisen R. 2010. Arthropod CYPomes illustrate the tempo and mode in P450 evolution. Biochim Biophys Acta. 1814(1):19-28. doi: 10.1016/j.bbapap.2010.06.012

5. Fokkens L, Guo, L., Dora, S., Wang, B., Ye, K., Sánchez-Rodríguez C., Croll, D. (2020). A Chromosome-Scale Genome Assembly for the Fusarium oxysporum Strain Fo5176 To Establish a Model Arabidopsis-Fungal Pathosystem. G3 (Bethesda). 10(10):3549-3555. doi: 10.1534/g3.120.401375

6. Guengerich, F. P. (2020). A history of the roles of cytochrome P450 enzymes in the toxicity of drugs. Toxicological Research, Table 2. https://doi.org/10.1007/s43188-020-00056-z

7. Keller, N. P. (2019). discovery. 17(3), 167-180. https://doi.org/10.1038/s41579-018-0121-1.Fungal

8. Kelly SL, Lamb DC, Baldwin BC, Corran AJ, Kelly DE. 1997. Characterization of Saccharomyces cerevisiae CYP61, sterol delta22-desaturase, and inhibition by azole antifungal agents. J Biol Chem. 272(15):9986-8.

9. Lysøe, E., Frandsen, R.J.N., Divon, H.H., Terzi, V, Orrù L, Lamontanara, A, Kolseth, A.K.,

10. Nielsen, K.F., Thrane, U. (2016). Draft genome sequence and chemical profiling of Fusarium langsethiae, an emerging producer of type A trichothecenes. Int J Food Microbiol, 221:29-36. doi: 10.1016/j.ijfoodmicro.2016.01.008

11. Ma, L., Geiser, D. M., Proctor, R. H., Rooney, A. P., Donnell, K. O., Trail, F., Gardiner, D. M., Manners, J. M., \& Kazan, K. (2013). Fusarium Pathogenomics. https://doi.org/10.1146/annurev-micro-092412-155650

12. Minerdi, D., Sadeghi, S. J., Pautasso, L., Morra, S., Aigotti, R., Medana, C., Gilardi, G., Lodovica, M., \& Gilardi, G. (2019). BBA - Proteins and Proteomics Expression and role of CYP505A1 in pathogenicity of Fusarium oxysporum $\mathrm{f}$. $\mathrm{sp}$. lactucae. BBA - Proteins and Proteomics, August, 140268. https://doi.org/10.1016/j.bbapap.2019.140268

13. Munkvold, G. P. (2017). Chapter 4 Fusarium Species and Their Associated Mycotoxins (Vol. 1542). https://doi.org/10.1007/978-1-4939-6707-0

14. Munkvold, G. P., Proctor, R. H., \& Moretti, A. (2021). Mycotoxin Production in. 1-30.

15. Nakayama N, Takemae A, Shoun H. 1996. Cytochrome P450foxy, a catalytically self-sufficient fatty acid hydroxylase of the fungus Fusarium oxysporum. J Biochem. 119(3):435-40. doi: 10.1093/oxfordjournals.jbchem.a021260.

16. Niehaus, E.M., Münsterkötter, M., Proctor, R.H., Brown, D.W., Sharon, A., Idan, Y., Oren-Young, L., Sieber, C.M., Novák, O., Pěnčík, A., Tarkowská, D., Hromadová, K., Freeman, S., Maymon, M., Elazar, M., Youssef, S.A., El-Shabrawy, E.S., Shalaby, A.B., Houterman, P., Brock, N.L., Burkhardt, I., Tsavkelova, E.A., Dickschat, J.S., Galuszka, P., Güldener, U., Tudzynski, B. (2016). Comparative "Omics" of the Fusarium fujikuroi Species Complex Highlights Differences in Genetic Potential and Metabolite Synthesis. Genome Biol Evol. 8(11):3574-3599. doi: 10.1093/gbe/evw259

17. Proctor RH, Brown DW, Plattner RD, Desjardins AE. 2003. Co-expression of 15 contiguous genes delineates a fumonisin biosynthetic gene cluster in Gibberella moniliformis. Fungal Genet Biol. 38(2):237-49. doi: 10.1016/s10871845(02)00525-x.

18. Proctor, R. H., Brown, D. W., Sharon, A., Niehaus, E., Mu, M., Freeman, S., Maymon, M., Elazar, M., Youssef, S. A., Elshabrawy, E. S. M., Shalaby, A. B. A., Houterman, P., Brock, N. L., \& Burkhardt, I. (2016). Comparative "Omics" of the Fusarium fujikuroi Species Complex Highlights Differences in Genetic Potential and Metabolite Synthesis “. 8(11), 3574-3599. https://doi.org/10.1093/gbe/evw259

19. Qhanya, L. B., Matowane, G., Chen, W., \& Sun, Y. (2015). Genome-Wide Annotation and Comparative Analysis of Cytochrome P450 Monooxygenases in Basidiomycete Biotrophic Plant Pathogens. 1-17. https://doi.org/10.1371/journal.pone.0142100

20. Rampersad, S. N. (2020). Pathogenomics and Management of Fusarium Diseases in Plants. i. 
21. Saha, P., Sarkar, A., Sabnam, N., Shirke, M. D., Mahesh, H. B., Nikhil, A., Rajamani, A., Gowda, M., \& Roy-barman, S. (2021). Comparative analysis of secondary metabolite gene clusters in different strains of Magnaporthe oryzae. May 2020, 1-9. https://doi.org/10.1093/femsle/fnaa216

22. Seepe, H. A., Amoo, S. O., Nxumalo, W., \& Adeleke, R. A. (2020). South African Journal of Botany Sustainable use of thirteen South African medicinal plants for the management of crop diseases caused by Fusarium species $\dot{A}$ An in vitro study. 130, 456-464. https://doi.org/10.1016/j.sajb.2020.01.038

23. Sello MM, Jafta M, Nelson DR, Chen W, Yu J-H, Parvez M, et al. 2015. Diversity and evolution of cytochrome P450 monooxygenases in Oomycetes. Sci Rep. 5: 11572 10.1038/srep11572

24. Shin, J., Kim, J., Lee, Y., \& Son, H. (2018). Fungal Cytochrome P450s and the P450 Complement (CYPome) of Fusarium graminearum. 76-91. https://doi.org/10.3390/toxins10030112

25. Syed K, Shale K, Pagadala NS, Tuszynski J. 2014. Systematic identification and evolutionary analysis of catalytically versatile cytochrome p450 monooxygenase families enriched in model basidiomycete fungi. PLoS One. 22;9(1):e86683. doi: 10.1371/journal.pone.0086683.

\section{Table}

Due to technical limitations, table 2 is only available as a download in the Supplemental Files section.

\section{Figures}




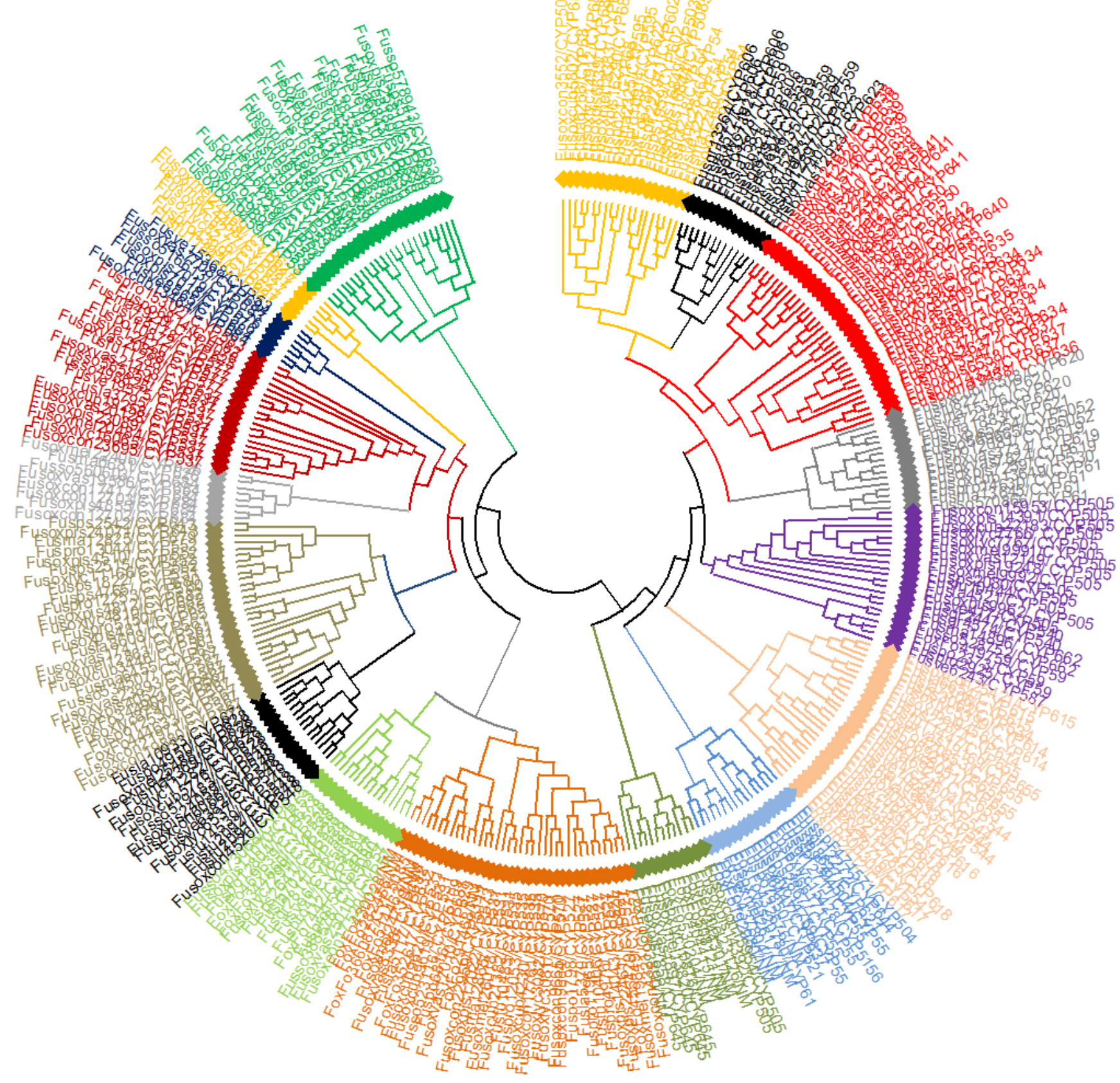

Figure 1

Evolutionary Relationship of Cytochrome P450 genes across seventeen selected Fusarium species which was inferred using MEGA X. 320 protein sequences were used. 

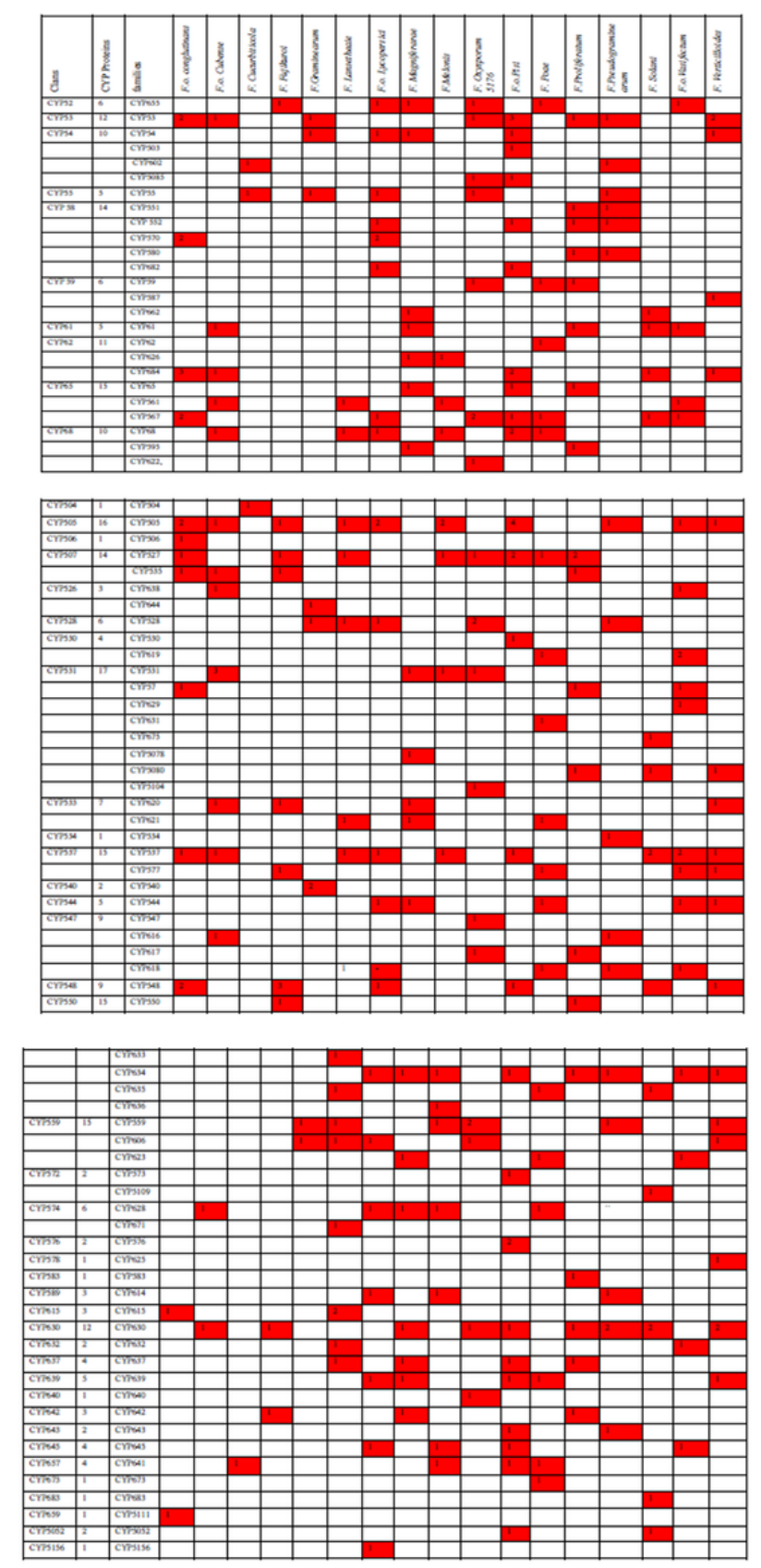

\section{Figure 2}

Distribution of identified families and clans across the seventeen selected Fusarium species 


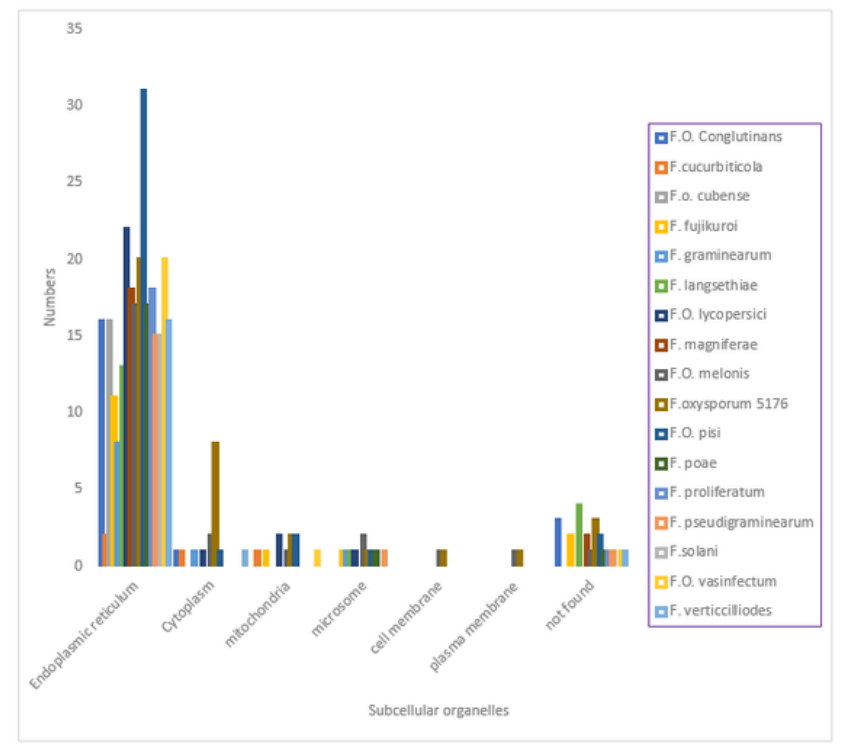

(a)

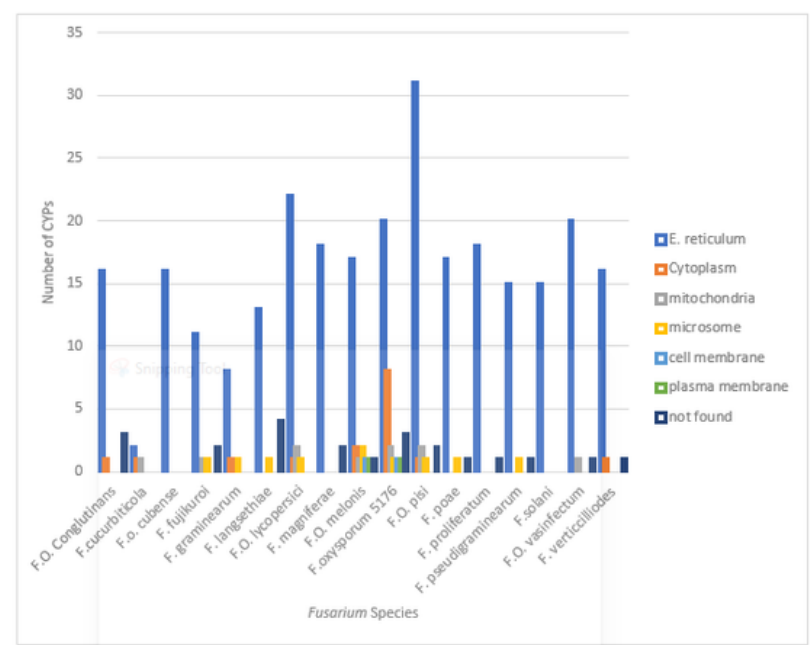

(b)

\section{Figure 3}

a: Predicted subcellular localization across seventeen selected Fusarium spp. b: Predicted subcellular localization across seventeen selected Fusarium spp. 


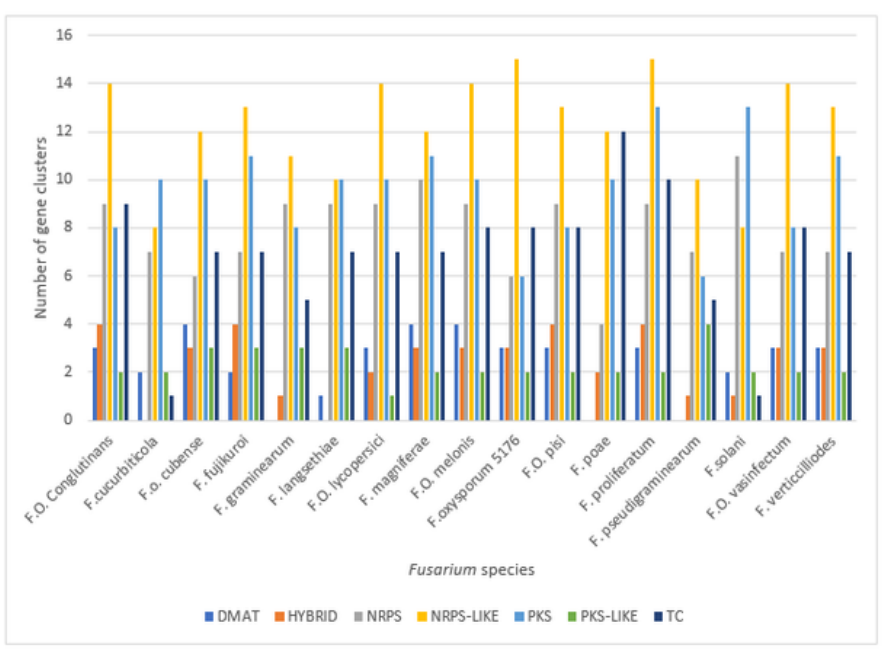

(a)

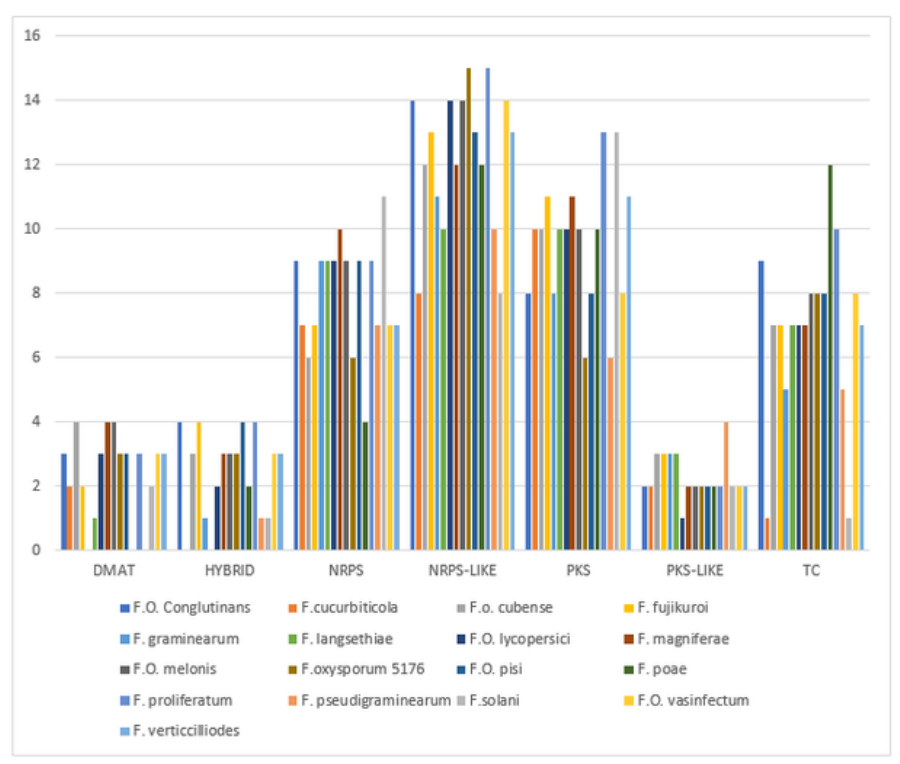

(b)

\section{Figure 4}

a: Distribution of Secondary Metabolism Gene clusters in each of the seventeen Selected Fusarium species DMATS: DiMethyl Allyl Tryptophan Synthase NRPS: Non-Ribosomal Peptide Synthetases NRPS-LIKE: Non-Ribosomal Peptide Synthetases-LIKE PKS: PolyKetide Synthases PKS-LIKE: PolyKetide Synthases-LIKE TC: Terpene Cyclases b: Distribution of Each Secondary Metabolism Gene Clusters Across Seventeen Selected Fusarium species DMATS: DiMethyl Allyl Tryptophan Synthase NRPS: Non-Ribosomal Peptide Synthetases NRPS-LIKE: Non-Ribosomal Peptide Synthetases-LIKE PKS: PolyKetide Synthases PKS-LIKE: PolyKetide Synthases-LIKE TC: Terpene Cyclases

\section{Supplementary Files}

This is a list of supplementary files associated with this preprint. Click to download. 
- Table.docx

Page 15/15 